\title{
RELEVÂNCIA SISTEMÁTICA DA MORFOLOGIA DE FRUTOS DOS GÊNEROS AMERICANOS DA TRIBO HIPPOMANEAE A. JUSS. EX BARTL. [EUPHORBIACEAE A. JUSS.]
}

\author{
SYSTEMATIC RELEVANCE OF THE MORPHOLOGY OF FRUITS OF AMERICAN GENERA OF HIPPOMANEAE A. \\ JUSS. EX BARTL. [EUPHORBIACEAE A. JUSS.]
}

RELEVANCIA SISTEMÁTICA DE LA MORFOLOGÍA DE LOS FRUTOS DE LOS GÉNEROS AMERICANOS DE
HIPPOMANEAE A. JUSS. EX BARTL. EUUPHORBIACEAE A. JUSS.]

Sarah Maria Athiê-Souza ${ }^{*}$

André Laurênio de Meloº

Marcos José da Silva ${ }^{3}$

\section{Resumo}

Margareth Ferreira de Sales ${ }^{4}$

Foi realizado um estudo morfológico de frutos e sementes de 12 gêneros Americanos da tribo Hippomaneae, objetivando avaliar a importância taxonômica dessas estruturas. Alguns caracteres são consistentes (deiscência do fruto, comprimento do estilete, septo interno do mericarpo, fenda oblíqua triangular na base dos carpídios, forma do ápice e das alas da columela, carpidióforo, arilo, presença e forma da carúncula e ornamentação da superfície da semente) e permitem a caracterização de cada gênero. Os resultados confirmam a importância taxonômica de frutos e sementes para os gêneros americanos de Hippomaneae. Descrições dos frutos e sementes de cada gênero e ilustrações são fornecidas.

Palavras-chave: Euphorbioideae, Gymnanthes, Microstachys, Sapium, Sebastiania.

\begin{abstract}
A morphology study of fruit and seed of 12 American genera in the tribe Hippomaneae is presented, aiming to evaluate the taxonomy value of these characters. Some characters are consistent (e.g., fruit dehiscence, style length, shape of the mericarp internal septum, occurrence mericarp with separate basal triangle, wings and columella apex shapes, presence or absence of carpidiophore, the presence of aril, presence and shape of the caruncle and surface ornamentation),

1 Universidade Federal da Paraíba, Centro de Ciências Exatas e da Natureza, Departamento de Sistemática e Ecologia, 58051-90o, João Pessoa, PB, Brazil; https://orcid.org/oooo-0oo2-6090-981X *Autor para correspondência: sarah_athie@yahoo.com.br

2 Unidade Acadêmica de Serra Talhada, Universidade Federal Rural de Pernambuco, 569oo-ooo, Serra Talhada, PE, Brazil; email: andrelaurenio@yahoo.com.br

3 Universidade Federal de Goiás, Instituto de Ciências Biológicas, 74001-970, Goiânia, GO, Brazil; email: marcos_agrorural@hotmail.com

4 Universidade Federal Rural de Pernambuco, 52171-9oo, Recife, PE, Brazil; email: mfsales65@hotmail. com, https://orcid.org/oooo-00o2-9587-1209
\end{abstract}


and characterize genera. The results confirm the taxonomic importance this structures for American genus Hippomaneae. In addition fruits and seeds descriptions of each genus and illustrations were given.

Key words: Euphorbioideae, Gymnanthes, Microstachys, Sapium, Sebastiania.

\section{Resumen}

Se presenta un estudio morfológico de frutos y semillas de 12 géneros americanos en la tribu Hippomaneae, con el objetivo de evaluar el valor taxonómico de estos caracteres. Algunos caracteres son consistentes (dehiscencia de la fruta, longitud del estilo, forma del septo interno del mericarpo, mericarpo de ocurrencia con triángulo basal separado, alas y formas de ápice de columela, presencia o ausencia de carpoforo, presencia de arilo, presencia y forma de la carúncula y ornamentación superficial), y caracterizan los géneros. Los resultados confirman la importancia taxonómica que esta estructura tiene para el género americano Hippomaneae. Además se dieron descripciones de frutas y semillas de cada género e ilustraciones.

Palabras clave: Euphorbioideae, Gymnanthes, Microstachys, Sapium, Sebastiania.

\section{INTRODUÇÃO}

A tribo Hippomaneae A. Juss. ex Bartl. (Euphorbiaceae, Euphorbioideae), com distribuição pantropical inclui 34 gêneros e cerca de 300 espécies (Athiê-Souza et al. 2015), e compreende um grupo de plantas com taxonomia complexa decorrente da uniformidade de caracteres vegetativos e pequena variabilidade floral, cujas delimitações vêm sofrendo alterações desde o seu estabelecimento até o presente (Esser 2001). Diferencia-se das demais tribos da subfamília Euphorbioideae pelas inflorescências tirsóides, espiciformes e bissexuais, brácteas florais biglandulares, botões florais inclinados, flores apétalas, sépalas pequenas e imbricadas e estames eretos (Scholz 1964; Esser 1999a).

A dificuldade em delimitar os gêneros dentro da tribo está refletida na intricada história da taxonomia de Hippomaneae, o que pode ser constatado nas diversas classificações propostas para a tribo (Müller 1866). Em especial, quando os tratamentos envolveram os limites entre Sebastiania Spreng., Microstachys A. Juss. e Gymnanthes Sw., os autores quase sempre foram discordantes principalmente porque deram maior peso a caracteres vegetativos e florais pouco consistentes. Gymnanthes e Microstachys já fizeram parte da circunscrição de Sebastiania e Sebastiania já constou da sinonímia de ambos os gêneros (Jussieu 1824; Müller 1863). Embora Microstachys tenha sido aceito por Müller (1866), quase sempre foi tratado como uma seção de Sebastiania (Müller 1873; Bentham e Hooker 1880; Pax 1890; Pax e Hoffmann 1912; Webster 1994). Por outro lado, Esser et al. (1997) restabeleceram Microstachys. No caso de Gymnanthes, Müller (1866) o incluiu na circunscrição de 
Sebastiania sect. Gussonia, enquanto que Pax e Hoffmann (1912) e Webster (1994) o consideraram-no como um gênero distinto de Sebastiania. Os gêneros Maprounea Aubl., Sebastiania e, especialmente, Sapium Jacq. são também historicamente relacionados à Stillingia Gard. ex L. porque podem ser facilmente confundidos no estádio vegetativo (Rogers 1951). No entanto, a persistência do carpidióforo em Stillingia o diferencia dos demais gêneros da tribo (Rogers 1951; Esser 1999; 2001; Athiê-Souza et al. 2014). Até recentemente, a morfologia dos frutos e sementes eram pouco utilizadas na delimitação de táxons em Hippomaneae, quando Kruijt (1996) e Esser (1999) passaram a reconhecê-los como estruturas importantes que poderiam fornecer informações relevantes sobre as afinidades intergenéricas na tribo. Esser et al. (1997) e Esser (2001; 2003) mencionaram que a presença ou ausência de fenda oblíqua triangular na base interna do carpídio, formato do ápice da columela, presença e forma das alas na columela, superfície da testa da semente e presença ou ausência de carúncula poderiam ser utilizados para a delimitação genérica em Hippomaneae.

Dessa forma, o objetivo deste artigo foi descrever a morfologia dos frutos e sementes de representantes americanos da tribo Hippomaneae e fornecer caracteres úteis para facilitar a diferenciação destes gêneros, principalmente aqueles com delimitação conflituosa.

\section{MATERIAL E MÉTODOS}

Foram analisados cerca de 3000 espécimes depositados nos seguintes herbários: ALCB, BHCB, BM, CEN, CEPEC, CESJ, EAC, EAN, ESA, ESAL, F, GUA, HEPH, HRCB, HUEFS, HUFU, HXBH, IAC, IAN, IBGE, ICN, INPA, IPA, K, LD, MACN, MBM, MEXU, MICH, MO, NY, PACA, PEL, PEUFR, R, RB, SMDB, SPF, TEPB, UB, UEC, UFMT, UFP, UFS, VIC, WU, XAL, acrônimos segundo Thiers (2017). Foram realizadas coletas em várias localidades do Nordeste brasileiro durante os estudos filogenéticos e morfológicos de Sebastiania (Athiê-Souza 2010), e em excursões posteriores, sendo os frutos conservados em álcool etílico a $70 \%$, seguindo as técnicas usuais em taxonomia (Mori et al. 1989). O material foi incorporado ao acervo do Herbário Professor Vasconcelos Sobrinho (PEUFR) da Universidade Federal Rural de Pernambuco (UFRPE). Foram analisados os caracteres de frutos e de sementes de 63 espécies americanas pertencentes a 12 gêneros de Hippomaneae (Tabela 1). Quando o material encontrava-se incompleto, indisponível ou danificado as informações sobre os frutos foram obtidas de coleções-tipo e bibliografia especializada (Pax e Hoffman 1912; Esser 1994; 2001). A terminologia empregada foi baseada em Radford et al. (1974), Roth (1977), Barroso et al. (1999) e Esser (1994; 1999a; 2001; 2003). 
Tabela 1. Lista de gêneros e espécies americanos pertencentes à tribo Hippomaneae analisados.

\begin{tabular}{ll}
\hline Gênero & Espécie \\
\hline Actinostemon Mart. ex Klotzsch & A. appendiculatus Jabl. \\
& A. concolor (Spreng.) Müll. \\
& A. klotzschii (Didr.) Pax \\
& A. schomburgkii (Klotzsch) Hochr. \\
& A. verticillatus (Klotzsch) Baill. \\
\hline Colliguaja Molina & Colliguaja brasiliensis Klotzsch. \\
\hline Gymnanthes Sw. & G. actinostemoides Müll. Arg. \\
& G. elliptica Sw. \\
& G. glabrata (Mart.) Govaerts \\
& G. guatemalensis Standl. \& Steyerm. \\
& G. klotzschiana Müll. Arg. \\
G. longipes Müll. Arg. \\
G. lucida Sw. \\
G. schottiana Müll. Arg. \\
G. serrata Baill. \\
\hline H. mancinella L. \\
\hline M. fistulifera Benth. \\
M. nitida Spruce ex Benth. \\
M. occidentalis Benth. \\
\hline M. guianensis Aubl. \\
\hline Mabromantentata (Mart. \& Zucc.) H.-J. Esser \\
M. corniculata (Vahl.) Griseb. \\
M. serrulata (Mart. \& Zucc.) Müll. Arg. \\
P. bilocularis (Watson) Esser \\
P. lottiae (McVaugh) A.L.Melo \& Esser \\
P. membranifolia (Müll. Arg.) Esser \& A.L. Melo \\
P. tuerckheimiana (Pax \& K. Hoffm.) A.L. Melo \& Esser \\
\hline
\end{tabular}




\begin{tabular}{|c|c|}
\hline Gênero & Espécie \\
\hline \multirow[t]{9}{*}{ Sapium Jacq. } & S. argutum (Müll. Arg.) Huber \\
\hline & S. ciliatum Hemsl. \\
\hline & S. glandulosum (L.) Morong. \\
\hline & S. haematospermum Müll. Arg. \\
\hline & S. lanceolatum (Müll. Arg.) Huber \\
\hline & S. marginatum Müll. Arg. \\
\hline & S. marmieri Huber \\
\hline & S. obovatum Klotzsch ex Müll. Arg. \\
\hline & S. sellowianum (Müll. Arg.) Klotzsch ex Baill. \\
\hline \multirow[t]{11}{*}{ Sebastiania Spreng. } & S. brevifolia (Klotzsch ex Müll. Arg.) Müll. Arg. \\
\hline & S. chahalana Lundell \\
\hline & S. glandulosa (Sw.) Müll. Arg. \\
\hline & S. jacobinensis (Müll. Arg.) Müll. Arg. \\
\hline & S. macrocarpa Müll. Arg. ex Müll. Arg. \\
\hline & S. obtusifolia (Kunth) Pax \& Hoffm. \\
\hline & S. pallens (Griseb.) Müll. Arg. \\
\hline & S. pavoniana (Müll. Arg.) Müll. Arg. \\
\hline & S. pteroclada (Müll. Arg.) Müll. Arg. \\
\hline & S. riparia Klotzsch ex Schrad. \\
\hline & S. subsessilis (Müll. Arg.) Pax \\
\hline Senefeldera Mart. & S. verticillata (Vell.) Croizat \\
\hline \multirow[t]{15}{*}{ Stillingia Gard. ex L. } & S. acutifolia (Benth.) Hemsl. \\
\hline & S. argutedentata Jabl. \\
\hline & S. bicarpelaris S. Wats. \\
\hline & S. dichotoma Müll. Arg. \\
\hline & S. linearifolia S. Wats. \\
\hline & S. loranthacea (Müll. Arg) Pax \\
\hline & S. oppositifolia Müll. Arg. \\
\hline & S. paucidentata S. Wats. \\
\hline & S. saxatilis Müll. Arg. \\
\hline & S. spinulosa Torr. \\
\hline & S. tenella (Pax \& K. Hoffm.) \\
\hline & S. trapezoidea Ule \\
\hline & S. treculiana (Müll. Arg.) I.M.. Johnst. \\
\hline & S. uleana Pax \& K. Hoffm. \\
\hline & S. zelayensis (Kunth) Müll. Arg. \\
\hline
\end{tabular}




\section{RESULTADOS}

Chave para identificação dos gêneros Americanos da tribo Hippomaneae.

1. Fruto drupáceo e indeiscente; lóculos 6 a 9 por fruto, semente sem carúncula

4. Hippomane

1. Fruto cápsula septífraga, loculicida; lóculos 3 por fruto, semente com ou sem carúncula

9. Sapium

2. Cápsula septífraga; semente com arilo avermelhado 2

2. Cápsula loculicida; semente sem arilo . .3 3. Porção basal do fruto persistindo como um carpidióforo lenhoso após a sua iscência

12. Stillingia

3. Porção basal do fruto não persistente, sem carpidióforo .4

4. Fruto com vértices dos lobos carenados 2. Colliguaja

4. Fruto com vértices dos lobos nunca carenados .5

5. Columela trifacetada; semente rugosa 11. Senefeldera

5. Columela claviforme, em forma de T, linear, trifacetada com ou sem alas; sementes lisas, foveoladas ou alveoladas .6

6. Fruto com septo interno dos mericarpos partidos em forma de C; columela claviforme, com excrescências carnosas aderidas, sem carúncula 10. Sebastiania

6. Fruto com septo interno dos mericarpos íntegros ou caducos; columela trifacetada, em forma de $\mathrm{T}$ ou linear, sem excrescências carnosas aderidas, com ou sem carúncula

7. Semente alveolada com carúncula caliptriforme recobrindo todo o seu ápice 6. Maprounea 7. Semente lisa ou foveolada, sem carúncula ou com carúncula nunca recobrindo todo o seu ápice .8

8. Fruto apiculado, corte na porção superior da valva em forma de triângulo; columela em forma de T 8. Pleradenophora 8. Fruto não apiculado, porção superior da valva sem projeção triangular; columela trifacetada ou linear, com ou sem alas ......9 9. Fruto piloso, com estilete maior que $1 \mathrm{~cm}$; columela trifacetada alada .5. Mabea 9. Fruto pubescente ou glabro, com estilete menor que $1 \mathrm{~cm}$; columela trifacetada ou linear sem alas ...10 10. Columela linear Actinostemon

10. Columela trifacetada . .11
11. Carúncula estipitada . Microstachys
11. Carúncula sem estípite .3. Gymnanthes 
1. Actinostemon Mart. ex Klotzsch, Arch. Naturgesch. $7(1):$ 184. 1841. Tipo: Actinostemon grandifolius Klotzsch. [?= A. concolor (Spreng.) Müll. Arg.]

Cápsula loculicida, globoide a trilobada, 0,7 $\times 0,6-0,8 \mathrm{~cm}$, castanho-clara, longamente pedicelada (pedicelo maior que $3 \mathrm{~cm}$ compr.), dorso dos carpídios liso ou corniculado (A. verticillatus com 2 cornículos espiniformes por carpelo), glabro a pubescente em $A$. verticillatus, septo interno íntegro, com fenda oblíqua triangular na base interna dos carpídios; columela persistente, linear, sem alas. Semente oblongoide, base truncada, ápice truncado com apículo proeminente, 5-6 × 5-6 mm, castanha com ou sem máculas marmorizadas, com carúncula, arilo ausente, testa lisa.

Material selecionado: A. appendiculatus Jabl. BRASIL. Alagoas: Coruripe, 2/X/1999, Lyra-Lemos \& Bayma 4454 (IPA, PEUFR). A. concolor (Spreng.) Müll. Arg. Rio de Janeiro: Restinga de Marambaia, 3o/I/1992, fl., fr., Araujo 9553 (GUA). São Paulo: Vinhedo, 23/XI/1994, fl., fr., Mendacolli et al. 689 (IAC). A. klotzschii (Didr.) Pax. Paraná: Cianorte, 25/VIII/1967, Hatschbach s.n (ESA 73001). A. schomburgkii (Klotzsch) Hochr. Mato Grosso: Rio Aripuanã, 5921'N, 10ํㅜ'ㄴ, 14.X.1973, fr., Berg et al. 18503 (INPA 41805). A. verticillatus (Klotzsch) Baill. Minas Gerais: Viçosa, 10/ XII/1934, fr., Kuhlman s.n (VIC 1493).

2. Colliguaja Molina, Sag. Stor. Nat. Chili: 158. 1782. Tipo: Colliguaja odorifera Molina

Cápsula loculicida, triangular ou trilobada, 0,5-0,7 $\times 0,7-0,8 \mathrm{~cm}$, castanha, curto-pedicelada (o,2-0,3 cm compr.), dorso dos carpídios liso, glabro, vértices dos lobos carenados, septo interno íntegro e inconspícuo, sem fenda oblíqua triangular na base interna dos carpídios; columela persistente, trifacetada, com alas. Semente ovoide a globoide, base arredondada, ápice obtuso, ca. $4 \times 2 \mathrm{~mm}$, castanha com máculas marmorizadas, sem carúncula, arilo ausente, testa lisa.

Material selecionado: $\boldsymbol{C}$. brasiliensis Klotzsch. BRASIL. Paraná: Bocaíuva do Sul, 29/VI/1972, fr., Hatschbach 29769 (MBM). Rio Grande do Sul: Santa Maria, 20/IX/1991, fl., fr., Jarenkow et al. 1897 (MBM).

3. Gymnanthes Sw., Prodr. Veg. Ind. Occ. 6: 95. 1788. Tipo: Gymnanthes elliptica Sw.

Fig. 1A-D.

Cápsula loculicida, globoide, oblongoide ou trilobada, o,4-1,6 $\times 0,3^{-1,6} \mathrm{~cm}$, castanha, longamente pedicelada (geralmente maior que 3,0 cm compr.), dorso dos carpídios liso ou corniculado ( $G$. schottiana e G. glabrata), glabro a pubescente em G. klotzschiana e G. longipes, septo interno íntegro, com fenda oblíqua triangular na base interna dos carpídios; columela persistente, trifacetada, sem alas. Semente

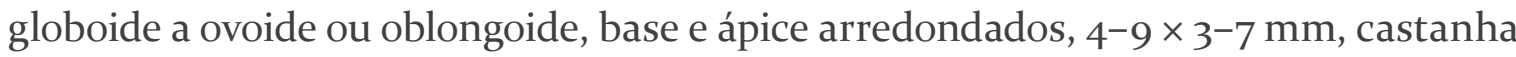
com ou sem máculas marmorizadas, com carúncula (G. actinostemoides, G. elliptica, G. glabrata, G. guatemalensis, G. longipes, G. lucida, G. schottiana e G. serrata), arilo ausente, testa lisa ou foveolada (G. schottiana). 
Material selecionado: G. actinostemoides Müll. Arg. MEXICO. Chiapas: Amatenango Del Valle, 05/IX/1966, fr., Ton 1109 (F). PANAMA. Cerro Jefe, s.data, fr., Valdespino et al. 284 (F). G. elliptica Sw. JAMAICA. Clarendon: Crofts Mountains, 30/IX/1912, fr., Harris 11224 (F). G. glabrata (Mart.) Govaerts. BRASIL. Bahia: Juçari, 02/II/1994, fr., Thomas et al. 10218 (CEPEC). Rio de Janeiro: São Pedro d'Aldeia, 13/X/1987, fr., Araujo \& L. Fisher 8247 (GUA). G. guatemalensis Standl. \& Steyerm. COSTA RICA. Limón: North of Puerto Viejo de Limon, 24/X/1976, fr., Hartshorn 1853 (F). GUATEMALA. Baja Verapaz: Niño Perdido, 15/XI/1977, fr., Lundell \& E. Contreras 21117 (F). G. klotzschiana Müll. Arg. ARGENTINA. Isla Martin García, III/1923, fr., Hamman s/n (MACN 27/2311). BRASIL. Rio de Janeiro: Cristal, s/data, fr., Jarenkow 2775 (ESA). São Paulo: Piracicaba, o6/XI/1992, fr., Ivanauskas s.n (ESA 8447). G. longipes Müll. Arg. MEXICO. Jalisco: Puerto Vallarta, 20/II/1993, fr., Castillo et al. 9944 (XAL). Queretaro: Jalpan, 13/III/1990, fr., Servín 7 (XAL). G. lucida Sw. MEXICO. Campeche: Hecelchakán, 29/XII/1985, fr., Chan 5453 (F). Quintana Roo: B. Juaréz, 05/XIII/1983, fr., Escalante 101 (MEXU). Veracruz: Axocuapan, 07/VIII/1983, fr., Robles 299 (XAL). G. schottiana Müll. Arg. BRASIL. Minas Gerais: Itutinga, 29/III/1990, fr., Oliveira-Filho et al. s.n (ESAL 10048). Rio Grande do Sul: Parque do Turvo, 31/X/1971, fr., Linderman et al. s.n (ICN 8907). G. serrata Baill. BRASIL. Minas Gerais: Carrancas, o8/IV/1989, Van den Berg s.n (ESAL 9510). Rio Grande do Sul: Porto Alegre, 05/I/1976, fr., Allem s.n (ICN 30459). São Paulo: Guarulhos, s/data, fr., Gandolfi et al. 5791 (ESA).

\section{Hippomane L., Sp. Pl.: 1191. 1753. Tipo: Hippomane mancinella L.}

Fig. 1 E.

Fruto drupáceo com 6 a 9 lóculos, indeiscente, elipsoide a globoide, amarelo quando maduro, curto pedicelado (o,6 cm compr.), dorso dos carpídios liso, glabro, septo interno e fenda oblíqua triangular ausentes; columela ausente. Semente elipsoide, variando de seis a nove por fruto, lóculos unisseminados, sem máculas marmorizadas, carúncula e arilo ausentes, testa lisa. As informações foram baseadas na literatura.

5. Mabea Aubl., Hist. Pl. Guiane 2:867. 1775. Tipo: Mabea piriri Aubl.

Fig. 1 F-G.

Cápsula loculicida, globoide a trilobada, 0,9-1,6 $\times 0,7-1,5 \mathrm{~cm}$, castanha a castanho-avermelhada, pedicelada (até 1,5 cm compr.), dorso dos carpídios liso, piloso, septo interno íntegro, com fenda oblíqua triangular na base dos carpídios, estilete maior que $1 \mathrm{~cm}$; columela persistente, trifacetada, alada. Semente oblongoide (M. fistulifera) a elipsoide ( $M$. nitida), base e ápice arredondados, o,6-1 $\times 0,4-0,8 \mathrm{~cm}$, castanha com ou sem máculas, com carúncula, arilo ausente, testa lisa.

Material selecionado: M. fistulifera Benth. BRASIL. Maranhão: São Pedro dos Cacetes, 16/VII/1976, fr., Tavares 1302 (PEUFR). M. nitida Spruce ex Benth. 
Amazonas: Mojuí, 25/XI/1999, fr., Mesquita et al. 212 (PEUFR). M. occidentalis Benth. Pernambuco: Recife, 11/II/1993, fr., Melo et al. s.n (PEUFR 15054).

6. Maprounea Aubl., Hist. Pl. Guiane 2:895. 1775. Tipo: Maprounea guianensis Aubl.

Fig. $1 \mathrm{H}$.

Cápsula loculicida, globoide, o,5-0,8 $\times 0,5-0,7 \mathrm{~cm}$, castanho-escura a castanho avermelhada, pedicelada (o,6-1,3 cm compr.), dorso dos carpídios liso, glabro, septos do mericarpo delgados geralmente caducos após a deiscência do fruto, sem fenda oblíqua triangular na base dos carpídios; columela persistente, trifacetada, com alas caducas em virtude da sua grande fragilidade. Semente globoide, base e ápice arredondados, 4-5 × 4-5 mm, enegrecida sem máculas marmorizadas, carúncula caliptriforme recobrindo todo o seu ápice, arilo ausente, testa alveolada.

Material selecionado: M. guianensis Aubl. BRASIL. Bahia: Serra da água de rega, 26/II/1971, fr., Irwin et al. 31146 (K). Goiás: Pirenópolis, X/1996, fr., Brina s.n (BHCB 35110). Maranhão: Serra do Penitente, 23/III/1978, fr., Assis 85 (RB). Mato Grosso: Margem da BR-8o, II/1978, fr., Passos 1107 (RB).

7. Microstachys A. Juss., Euph. Gen.: 48. 1824. Tipo: Microstachys bicornis A. Juss. [= Microstachys corniculata (Vahl) Griseb.]

Fig. 1I-L.

Cápsula loculicida, oblongoide a globoide, 0,4-0,7 $\times 0,4^{-0,5} \mathrm{~cm}$, castanha, geralmente pedicelada $(0,2-0,3 \mathrm{~cm})$, dorso dos carpídios corniculados, variando de dois a muitos por carpelo, ligeiramente pubescente ( $M$. serrulata e $M$. corniculata), septo íntegro após a deiscência do fruto, com fenda oblíqua triangular na base dos carpídios; columela persistente, trifacetada, sem alas. Semente oblongoide, base arredondada, ápice truncado, 3-4 × 2-4 mm, castanho-esverdeada a enegrecida com ou sem máculas marmorizadas, com carúncula disciforme e estipitada, arilo ausente, testa foveolada.

Material selecionado: M. bidentata (Mart. \& Zucc.) H.-J. Esser BRASIL. Bahia: Piatã, 24/II/1994, fr., Sano et al. 14495 (ESA). Mato Grosso: s/local, s/data, fr., Godinho 4002 (UFMT). M. corniculata (Vahl.) Griseb. Bahia: Jacobina, 10/ IX/196o, fr., Costa s.n (ALCB 2293). Pernambuco: Buíque, 07/VI/2011, fl., fr., Souza et al. 17 (PEUFR). São Paulo: Picinguaba, 27/III/1993, fr., Romera et al. 20 (PEUFR). M. serrulata (Mart. \& Zucc.) Müll. Arg. Distrito Federal: s/local, 12/III/1981, fr., Kirkbride Jr. 3994 (HRCB). Minas Gerais: Paraopeba, 12/III/2002, fr., Valente \& M. Neto 950 (VIC).

8. Pleradenophora H-J. Esser., Gen. Euphorb. 352-370. 2001. Tipo: Pleradenophora tuerckheimiana (Pax \& K. Hoffm.) A.L.Melo \& Esser.

Fig. $1 \mathrm{M}$.

Cápsula loculicida, globoide a trilobada com apículo, 0,6-1,5 × 0,7-1,5 cm, castanha, pedicelo séssil a subséssil, dorso dos carpídios liso, glabro, septo interno íntegro, com fenda oblíqua triangular na base interna dos carpídios e corte na porção 
superior da valva em forma de triângulo; columela persistente em formato de T, com alas. Semente globoide a ovoide, base e ápice arredondados, 4-7 $\times$ 4-7 mm, castanha a enegrecida sem máculas marmorizadas, carúncula e arilo ausentes, testa foveolada.

Material selecionado: P. tuerckheimiana (Pax \& K. Hoffm.) A.L. Melo \& Esser. BELIZE. Cayo: Las Cuevas, 29/III/1973, fr., Croat 23549 (XAL). GUATEMALA. Peten: San Luis, 29/IV/1976, Ventur 233 (F). HONDURAS. s/local, VI/1933, fr., Lundell 4130 (F). MEXICO. Campeche: Escarcega, 1966, Chavelas \& J. Pérez 839 (F).

9. Sapium Jacq., Enum. Syst. Pl.: 9. 1760, nom. cons. [non Sapium P. Browne, Civ. Nat. Hist. Jamaica 2: 338. 1756]. Tipo: Sapium aucuparium Jacq., nom. illeg. [= Sapium glandulosum (L.) Morong.]

Fig. $1 \mathrm{~N}-\mathrm{Q}$.

Cápsula septífraga, ovoide (S. lanceolatum) a trilobada (S. glandulosum), o,61,1 $\times 0,7-1,2 \mathrm{~cm}$, castanha, pedicelada (1,0-1,5 cm compr.), dorso dos carpídios liso, glabro, sem septo interno nas valvas, sem fenda oblíqua triangular na base interna dos carpídios; columela persistente, trifacetada, alada. Semente oblongoide a ovoide, base truncada, ápice arredondado levemente apiculado, o,8-1 × 0,7-0,8 cm, castanha sem máculas marmorizadas, carúncula ausente, completamente recoberta por arilo avermelhado, testa lisa.

Material selecionado: S. argutum (Müll. Arg.) Huber. BRASIL. Alagoas: Mata Grande, 20/XII/1974, fl., Andrade-Lima 7788 (IPA). S. ciliatum Hemsl. Pará: beira da estrada Santarém-Cuiabá, entre km 45 e 48, lado direito, 1/IV/1974, fl., fr., J. Medeiros \& L. R. Marinho 67 (IAN). S. glandulosum (L.) Morong. Alagoas: Murici, Serra do Ouro, $9^{\circ} 13^{\prime}$ S, 3350' O, 12/IV/2008, fr., Pinheiro et al. 398 (RB). S. haematospermum Müll. Arg. Mato Grosso do Sul: Corumbá, 23/X/2008, fl., fr., Caxambu et al. 2304. S. marginatum Müll. Arg. Distrito Federal: Lago Sul, 29/IX/2009, fl., fr., Silva et al. 840 (HEPH). S. marmieri Huber. Acre: Cruzeiro do Sul, Alto Rio Juruá, Tapuama, $9^{\circ} 12^{\prime}$ S, 72 ${ }^{\circ} 14$ ' O, 14/III/1992, fr., Ferreira et al. 10814 (INPA);S. lanceolatum (Müll. Arg.) Huber. Pernambuco: Altinho, 25/III/2007, fr., Nascimento \& J.G. Melo 74 (PEUFR). S. obovatum Klotzsch ex Müll. Arg. Ceará: Mulungu, 12/III/2003, fr., Silveira \& R. F. Oliveira 941 (EAC). S. sellowianum (Müll. Arg.) Klotzsch ex Baill. Minas Gerais: Serra do Espinhaço, 26/I/1971, fr., Irwin et al. 29179 (UB).

10. Sebastiania Spreng., Neue Entdeck Pflanzenk. 2: 118, pl. 3. 1821. Tipo: Sebastiania brasiliensis Spreng.

Fig. 1R-S.

Cápsula loculicida, triangular a trilobada, 0,5-2,6 $\times 0,6-2,3 \mathrm{~cm}$, castanha, pedicelo subséssil, dorso dos carpídios frequentemente liso, às vezes epicarpo muricado em $S$. macrocarpa, glabro, septo interno com corte em forma de C, sem fenda oblíqua triangular na base interna dos carpídios; columela persistente, claviforme, alada, normalmente com excrescências inteiras exceto em $S$. macrocarpa com excrescências multífida. Semente globoide, ovoide, elipsoide ou obovoide, base arredondada, apiculada ou truncada, ápice arredondado ou apiculado, 3-8 $\times$ 
2-6 mm, castanha ou enegrecida com (S. macrocarpa, S. obtusifolia, S. pavoniana e $S$. riparia) ou sem máculas marmorizadas, carúncula e arilo ausentes, testa lisa, semente recoberta por excrescências apenas em $S$. macrocarpa.

Material selecionado: S. brevifolia (Klotzsch ex Müll. Arg.) Müll. Arg. BRASIL. Bahia: Umburanas, 10/IV/1999, fr., Queiroz et al. 5236 (HUEFS, PEUFR). Ceará: Tianguá, 24/III/1979, fr., Fernandes \& F. J. A. Matos s.n (PEUFR 40269). S. chahalana Lundell. GUATEMALA. Alta Verapaz: Chahal, 16/X/1968, fl., fr., Contreras 7948 (Isótipo F). S. glandulosa (Sw.) Müll. Arg. BELIZE. Orange Walk District: between London and Carmelita, 20/III/1987, fr., Davidse \& A. E. Brant 32828 (F). GUATEMALA. Petén: Dos Lagunas, 14/V/1969, fl., fr., Contreras 8544 (F). MEXICO. Quintana Roo: Cancún, 04/VIII/1987, fl., fr., Little Jr. 40310 (F). $S$. jacobinensis (Müll. Arg.) Müll. Arg. BRASIL. Bahia: Jacobina, 1841, fr., Blanchet 3428 (Holótipo NY, Isótipo F). Pernambuco: Brejo da Madre de Deus, 18/III/1992, fl., fr., Guedes 70 (IPA, PEUFR). S. macrocarpa Müll. Arg. ex Müll. Arg. BRASIL. Bahia: Livramento do Brumado, 17/IV/1991, fr., Lewis \& S. M. M. Andrade 2001 (UB). Paraíba: Areia, 26/V/1978, fr., Andrade-Lima 78-8429 (IPA). Pernambuco: Serra Talhada, 26/ II/2010, fr., Souza \& A. L. Melo o4 (PEUFR). Rio Grande do Norte: Açu, 26/II/1987, fr., Ferreira 69 (IPA). S. obtusifolia (Kunth) Pax \& Hoffm. PERU. Ancash: Huyalas, 8/V/1991, fr., Alban 6389 (F). Cajamarca: Valle de Cajamarca, 10/IV/1984, fl., fr., Vega 3339 (F). La Libertad: Santiago de Chuco, 13/VI/1984, fr., Sagástegui et al. 11792 (F). S. pallens (Griseb.) Müll. Arg. CUBA. Arroyo Trinitaria: Trinidad Mountains, o7/ III/1910, fr., Britton \& E. G. Britton 5164 (F). Santa Clara: Buenos Aires, 20/III/1931, fr., Jack 8222 (F). S. pavoniana (Müll. Arg.) Müll. Arg. COSTA RICA. Guanacaste: Parque Nacional Santa Rosa, o8/X/1994, fr., Cascante \& G. Vargas 306 (F). MEXICO. Chamela: La Huerta, 25/VII/1986, fr., Lott \& T. H. Atkinson 2818 (F). Puebla: Jolalpan, 19/VI/1984, fl., fr., Macías 67 (XAL). Veracruz: Corral de Las Piedras, V/1904, fr., Purpus 2425 (F). S. pteroclada (Müll. Arg.) Müll. Arg. BRASIL. Rio de Janeiro: Restinga de Marambaia, 17/III/1994, fr., Araújo 9975 (GUA). S. riparia Klotzsch ex Schrad. BRASIL. Paraíba: Itabaiana, 26/XI/1971, fl., fr., Andrade-Lima et al. 1071 (IPA, PEUFR). Sergipe: Canindé de São Francisco, 25/IX/20oo, fr., Coelho \& R. Silva 446 (PEUFR). S. subsessilis (Müll. Arg.) Pax. ARGENTINA. Misiones: San Ignácio, 14/III/1952, fr., Montes 15609 (LD). PARAGUAI: Campo Apepu am Tapiraguay-Flusse, s. data, fr., Hassler 4350 (Isótipo BM).

11. Senefeldera Mart., Flora 24 Beibl. 2: 29.1841. Tipo: Senefeldera multiflora Mart. [= Senefeldera verticillata (Vel.) Croizat].

Cápsula loculicida, globoide a trilobada, $0,7-0,9 \times 1,1-1,3 \mathrm{~cm}$, verdes, pedicelada (o,4-o,6 cm compr.), dorso dos carpídios liso, glabro, septo interno íntegro, com fenda oblíqua triangular na base interna dos carpídios; columela persistente, trifacetada, alada. Semente ovoide, 3-7 × 3-6 mm, castanha, com máculas marmorizadas, com carúncula, arilo ausente, testa rugosa. 
Material selecionado: $\boldsymbol{S}$. verticillata (Vell.) Croizat. BRASIL. Alagoas: Coimbra, 07/V/2002, fr., Oliveira s.n (UFP 36803). Pernambuco: São Vicente Férrer, 29/I/1999, fr., Ferraz et al. 595 (PEUFR).

12. Stillingia Gard. ex L., Mant. 19. 1767. Tipo: Stillingia sylvatica Gard. ex L.

Fig. 1T-U.

Cápsula loculicida, globoide a trilobada, o,4-0,9 $\times 0,3-0,8 \mathrm{~cm}$, castanha, curtamente pedicelado (até 1,o cm compr.), dorso dos carpídios liso, glabro, septo interno íntegro e sem fenda oblíqua triangular na base interna dos carpídios; columela às vezes caduca, trifacetada, alada, com carpidióforo persistente, o qual é caracterizado pela base dos carpídios triangular e triconiforme. Semente elipsoide, oblongoide a ovoide, base e ápice arredondados, 3-4,5 × 2,5-4,3 $\mathrm{mm}$, cinérea a enegrecida com ( $S$. dichotoma e S. saxatilis) ou sem (p.ex. S. acutifolia, S. argutedentata, S. bicarpellaris, S. oppositifolia, $S$. trapezoidea e $S$. uleana) máculas marmorizadas, carúncula inserida em uma depressão ventral, arilo ausente, testa lisa (S. oppositifolia) ou (S. bicarpellaris, S. dichotoma, S. saxatilis, S. trapezoidea e S. uleana).

Material selecionado: $\boldsymbol{S}$. acutifolia (Benth.) Hemsl. GUATEMALA. Chimaltenango: West of Patzum, 26/I/1947, fr, Williams \& A. R. Molina 11844 (F, MO). S. argutedentata Jabl. BRASIL. Espírito Santo: Nova Venécia, serra de Baixo, Pedra da Torre, 18/II/20o8, fl., fr., Fontana et al. 4838 (MBML). S. bicarpelaris S. Wats. MEXICO. Aguascalientes: Calvillo, rancho Media Luna, $21^{\circ} 47^{\prime} \mathrm{N}, 102^{\circ} 49^{\prime} \mathrm{W}$, 26/VII/1996, fl., fr., Provance 1667 (IEB). S. dichotoma Müll. Arg. - BRASIL. Rio de Janeiro: Morro de São João, o1/XI/1970, fr., Carautá 1247 (GUA). S. linearifolia S. Wats. ESTADOS UNIDOS. Arizona: Mohave, 7 miles south of Yucca, 12/IV/1947, fl., fr., Darrow \& Gould 3782 (F). Stillingia loranthacea (Müll. Arg) Pax. BRASIL. Bahia: Morro do Chapéu, 17/VI/2011, fl., fr., Souza et al. 46 (PEUFR). S. oppositifolia Baill. ex Müll. Arg. Rio Grande do Sul: Bento Gonçalves, 10/X/1998, fl., fr., Pedralli 101 (ICN). S. paucidentata S. Wats. ESTADOS UNIDOS. California: 1897, fl., fr., Purpus 5492 (K, MO, P). S. saxatilis Müll. Arg. BRAZIL. Minas Gerais: Datas, ca. 15 km S of Diamantina, 05/II/1972, fl., fr., Anderson et al. 35517 (MO, UB). S. spinulosa Torr. ESTADOS UNIDOS. Arizona: Yuma, 05/VI/1932, fl., fr., Clark 4937 (G). $\boldsymbol{S}$. tenella (Pax \& K. Hoffm.). ARGENTINA. Manuel Belgrano, $24^{\circ} 14^{\prime} \mathrm{S}, 65^{\circ} \mathrm{O} 4^{\prime} \mathrm{W}, 14 /$ XI/2002, fr., Zuloaga et al. 7655 (MO). S. trapezoidea Ule. BRAZIL. Pernambuco: Buíque, 07/VI/2011, fr., Souza et al. 15 (PEUFR). S. treculiana (Müll. Arg.) I.M.. Johnst. ESTADOS UNIDOS. Texas: Bexar, San Antonio, 29/XI/1911, fl., fr., Clemens \& Clemens 515 (MO). S. uleana Pax \& Hoffm. Bahia: Serra da Água de Rega, 24/II/1971, fl., fr., Irwin et al. 30968 (UB). S. zelayensis (Kunth) Müll. Arg. EL SALVADOR. Morazán: Arambala, $13^{\circ} 55^{\prime} \mathrm{N}, 88^{\circ} 6$ 'W, 16/X/2003, fr., Monterrosa \& Carbalho 769 (F). 


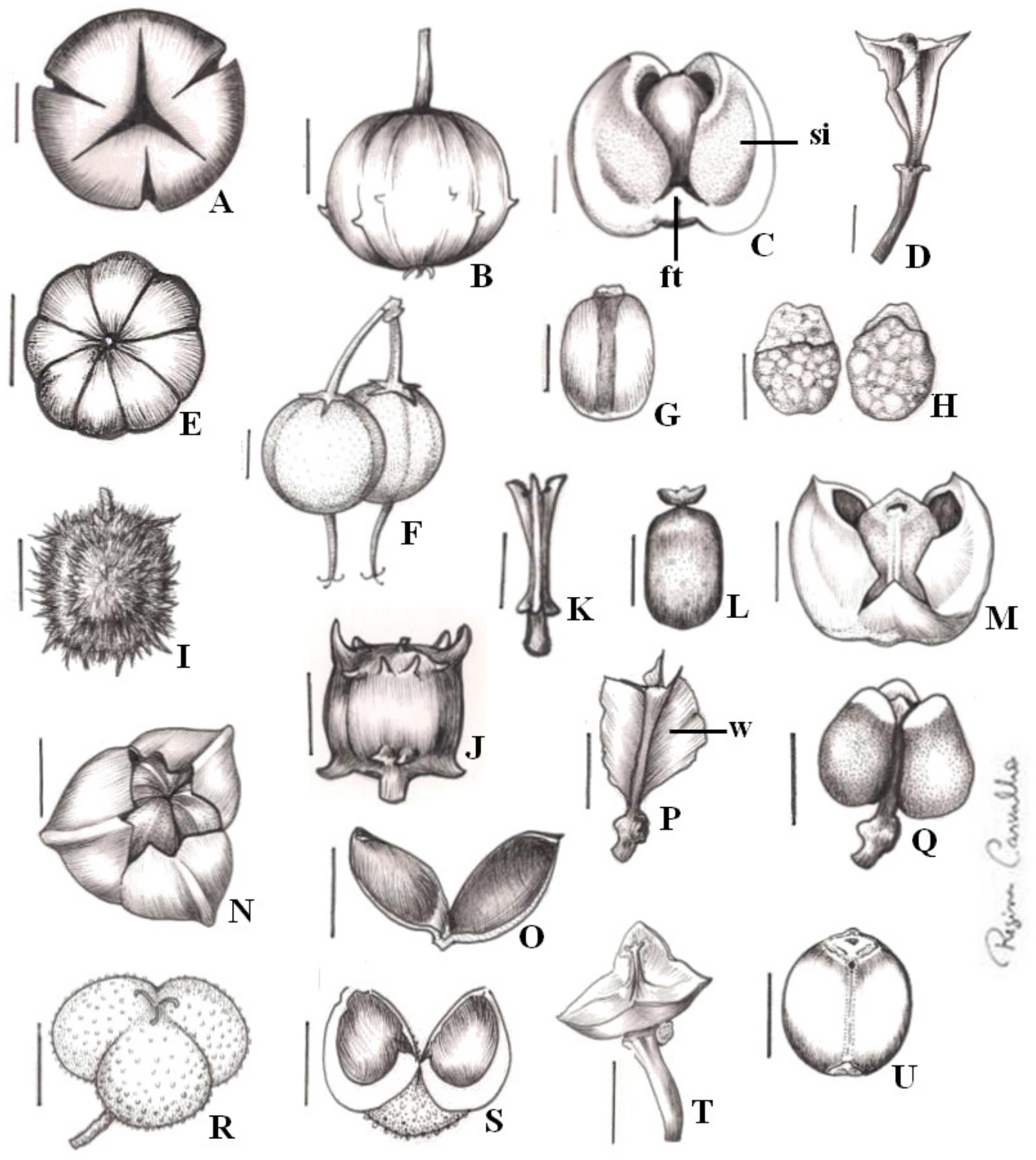

Figura. 1. A-D. Gymnanthes Sw. A, C-D. Gymnanthes klotzschiana Müll. Arg. (Ivanauskas ESA 8447), A. Cápsula loculicida; C. Mericarpo com fenda triangular e septo interno íntegro; D. Columela trifacetada sem alas; B. G. schottiana Müll. Arg. (Oliveira-Filho et al. ESAL 10048), fruto corniculado. E. Hippomane mancinela L., drupa 6-9 carpelar (adaptado da literatura). F-G. Mabea occidentalis Benth. (Melo et al. PEUFR 15054), F. Fruto com longo estilete; G. Semente carunculada. H. Maprounea guianensis Aubl. (Irwin et al. 31146), semente alveolada com carúncula caliptriforme. I-L. Microstachys. I. Microstachys corniculata (Vahl.) Griseb. (Romera et al. 2o), fruto corniculado; J-L. M. serrulata (Mart. \& Zucc.) Müll. Arg. (Valente E M. Neto 950), fruto corniculado, K. Columela trifacetada sem alas; L. Semente com carúncula estipitada; M. Pleradenophora tuerckheimiana (Pax \& K. Hoffm.) A.L.Melo \& Esser, mericarpo com fenda triangular e com corte na porção superior da valva em forma de triângulo (Lundell 4130). N-Q. Sapium Jacq. N. S. lanceolatum (Müll. Arg.) Huber. (Nascimento E Melo 74), cápsula septífraga; O-Q. Sapium glandulosum (L.) 
Morong. (Souza \& Melo o3); O. Ausência de septo interno nas valvas; P. Columela;.Q.

Semente com arilo; R-S. Sebastiania macrocarpa Müll. Arg. ex Müll. Arg. (Souza E A.L. Melo 04), R. Fruto muricado; S. Mericarpo sem fenda triangular e septo interno das valvas partido em forma de C. T-U. Stillingia dichotoma Müll. Arg. (Carauta 1247) T. Carpidióforo; U. Semente carunculada. Abreviações: w. alas; ft. mericarpo com triângulo basal separado; si. septo interno. Escalas em A-F, H-M, R-U - 2 mm; G - 2,5 mm; N-Q -5 mm.

\section{DISCUSSÃO}

Tipos de fruto - os gêneros estudados apresentam frutos do tipo cápsula 3-locular. No entanto, na tribo Hippomaneae são observados ainda frutos drupáceos e indeiscentes nos gêneros Hippomane (Esser 1999a; 2003; 2001) e Balakata Esser (Esser, 1999a). Em Sapium e Omalanthus (Geiseler) Pax podem ser encontradas cápsulas 2-loculares (Kruijt 1996; Esser 1997). Segundo Dilcher e Manchester (1988), a espécie fóssil Crepetocarpon perkinsii (Berry) Dilcher \& Manchester, identificada como pertencente à Hippomaneae, compartilha características dos frutos com H. mancinella, tais como indeiscência, número de carpelos superior a três, óvulos anátropos e uma semente por lóculo.

Deiscência - nos gêneros estudados, as cápsulas apresentam deiscência regular loculicida, dividindo-se em três carpídios, os quais se dividem posteriormente em duas valvas (Roth 1977; Esser, 1999a; Esser, 2003), exceto em Sapium que apresenta cápsula septífraga. Neste gênero, o endocarpo cartáceo, separa-se em três porções bífidas, na parte apical, sendo que as valvas ao se desprenderem da coluna central já estão inteiramente abertas e expondo as sementes (Barroso et al., 1999). No entanto, deiscência irregular ocorre em frutos de Falconeria Royle (Esser, 1999a).

Superfície do fruto - foram observados frutos com superfície lisa, muricada (Sebastiania macrocarpa) ou com protuberâncias corniculadas em algumas espécies dos gêneros Actinostemon, Gymnanthes, Microstachys e Sapium. No presente trabalho, apenas Actinostemon verticillatus, Gymnanthes glabrata, G. schottiana, Microstachys bidentata, M. corniculata, M. serrulata, Sapium glandulosum e $S$. lanceolatum apresentaram cornículos. De acordo com Esser (2003) a presença de cornículos nos frutos dessas espécies poderia indicar uma possível adaptação à epizoocoria ou estar relacionada a uma estratégia de proteção contra herbivoria em frutos autocóricos. Entretanto, não são observados estudos direcionados à biologia reprodutiva ou ontogenia das espécies com cornículos que esclareçam esta questão.

Fenda oblíqua triangular na base interna dos carpídios - este caráter é útil para separar dois grupos dentro da tribo: um com a fenda e o outro sem esta estrutura. O grupo com fenda foi representado por Actinostemon, Gymnanthes, Mabea, Microstachys, Pleradenophora e Senefeldera. No entanto, na tribo os gêneros Ditrysinia Raf., Grimmeodendron Urb., Sclerocroton Hochst., Senefelderopsis Steyerm., Dendrothrix Esser, Shirakiopsis Esser e Rhodothyrsus Esser também apresentam essa 
estrutura (Esser 1993; 1994; 1995; 1997; 1999a; 1999b; 2001; 2002; Kruijt 1996; Esser et al. 1997). O grupo sem a fenda é composto por Colliguaja, Maprounea, Sapium, Sebastiania e Stillingia. Vale salientar que a ausência deste caráter em Sebastiania pode ser utilizada para diferenciá-lo de gêneros que são morfologicamente relacionados, e que já fizeram parte da sua circunscrição como Ditrysinia, Gymnanthes, Microstachys e Pleradenophora (Melo 2006).

Arilo - Sapium possui as sementes inteiramente recobertas por uma camada carnosa avermelhada, que o distingue dos demais gêneros da tribo. Kruijt (1996) e Esser (1999) a referem como arilo. No entanto, Barroso et al. (1999) observam que essa camada carnosa seria uma sarcotesta. O termo sarcotesta é atribuído para as camadas produzidas em parte ou em toda a superfície do tegumento da semente (Corner 1976; Niembro 1988; Werker 1997). Entretanto, é mantido o termo arilo por se desconhecer a origem desta estrutura. Omalanthus e Falconeria também apresentam sementes recobertas por arilo, mas nestes casos, de coloração branca ou cinérea (ESSER 1997; 1999a).

Carúncula - está presente nos gêneros Actinostemon, Gymnanthes, Mabea, Maprounea, Microstachys e Stillingia. Segundo Corner (1976), a carúncula consiste em uma estrutura carnosa presente na extremidade micropilar da semente que se desenvolve na parte apical do integumento externo e forma o arilo exostomal ou arilóide. A carúncula na maioria dos gêneros é pequena e varia de subreniforme a largamente obdeltóide. Em Microstachys é disciforme e estipitada e em Maprounea é grande e caliptriforme recobrindo todo o ápice da semente, corroborando com as afirmações de Barroso et al. (1999) e Esser (2001). Segundo Esser (2001), algumas espécies de Excoecaria, Sebastiania e Spirostachys apresentam excrescências semelhantes às carúnculas (uma para cada lóculo), situadas acima da inserção da semente, que permanecem na columela após a deiscência do fruto e queda das sementes. No entanto, Melo (2006) afirma que em Sebastiania esta estrutura surge na região central do fruto (columela), acima do ápice da semente, embasado na análise de diversos frutos em diferentes fases de desenvolvimento, e por isso não poderia ser considerada uma carúncula.

Columela - esta estrutura pode ser considerada um dos principais caracteres que contribuem para a distinção dos táxons em Hippomaneae. A columela pode ser alada ou não. Quando alada, a ala é uma membrana fina constituída por parte do tecido interno dos septos, que permanece após a deiscência e queda dos carpídios. A columela pode ser: lineariforme, em Actinostemon; trifacetada, em Colliguaja, Gymnanthes, Mabea, Maprounea, Microstachys, Sapium, Senefeldera e Stillingia; com ala em forma de T, em Pleradenophora e claviforme, em Sebastiania.

Em Sebastiania a columela possui três excrescências geralmente inteiras, que não recobrem as sementes jovens, mas em $S$. macrocarpa estas são bem desenvolvidas e multífidas, recobrindo completamente as sementes nos frutos imaturos. A forma da columela é um caráter que pode ser usado também na distinção entre os gêneros Actinostemon e Gymnanthes, cuja delimitação genérica quase sempre foi discordante entre os autores que utilizaram caracteres vegetativos e florais. Nesse sentido, Pax 
e Hoffmann (1912) os diferenciaram com base nas sépalas e número de estames e Esser (2001) na presença de catáfilos recobrindo os botões florais e inflorescências em Actinostemon. Analisando exemplares herborizados destes táxons percebe-se que estes caracteres não são facilmente observados e não suportam a distinção entre os gêneros. Entretanto, a columela contribui de forma efetiva para a segregação dos dois gêneros, sendo que em Actinostemon ela é linear e trifacetada em Gymnanthes.

Carpidióforo - Stillingia apresentou uma peculiaridade entre os gêneros estudados, por possuir columela com a base lenhosa e tricórnea. Essa estrutura tem sido denominada como ginobase (Rogers 1951), carpóforo (Govaerts et al. 200o) ou carpidióforo (Esser 1999a; 2001). Esser et al. (1997) observaram em um estudo cladístico que Stillingia é proximamente relacionada a Sapium. Estes dois gêneros podem ser facilmente confundidos em estádio vegetativo, mas são diferenciados com base na presença de carpidióforo e ausência de arilo nas sementes em Stillingia.

\section{CONCLUSÃO}

Os resultados obtidos neste estudo mostram que os atributos morfológicos de frutos e sementes são úteis para a delimitação dos gêneros da tribo Hippomaneae, em virtude de serem consistentes nos espécimes analisados. Nas últimas décadas, especialistas em gêneros da tribo (Kruijt 1996; Esser 1999a; 2003; Melo 2006) vêm evidenciando a importância dos frutos e sementes para a distinção intergenérica. Entretanto, até o presente momento, nenhum estudo que abordasse os representantes de Hippomaneae com essa abordagem havia sido realizado. Os atributos tratados no presente trabalho são especialmente viáveis para distinguir gêneros historicamente relacionados, como Actinostemon, Sebastiania, Gymnanthes e Microstachys.

\section{AGRADECIMENTOS}

Ao Conselho Nacional de Desenvolvimento científico e tecnológico ( $\mathrm{CNPq}$ proc. 132124/2009-2) pela concessão da bolsa de mestrado à primeira autora, ao Programa de Pós-Graduação em Botânica (PPGB) da Universidade Federal Rural de Pernambuco, aos curadores dos herbários pelo empréstimo das exsicatas e à ilustradora Regina Carvalho.

\section{REFERÊNCIAS}

Athiê-Souza SM. 2006. Estudos morfológicos e filogenéticos de Sebastiania Spreng. (Hippomaneae, Euphorbiaceae). (Dissertação de Mestrado). Universidade Federal Rural de Pernambuco, Recife, PE. 
Athiê-Souza SM, Melo AL, Sales MF. 2014. Reinstatement and Lectotypification of Stillingia loranthacea (Euphorbiaceae), avulnerablespecies from Chapada Diamantina, Bahia (Brazil), and a new circumscription of Stillingia saxatilis. Systematic Botany 39: 510-516.

Athiê-Souza SM, Melo AL, Silva MJ, Oliveira LSD, Sales MF. 2015. Gradyana (Euphorbiaceae): a New Genus from Northeastern Brazil. Systematic Botany 40: 527-533.

Barroso GM, Morrim MP, Peixoto AL, Ichaso CLF. 1999. Frutos e Sementes. Morfologia Aplicada à Sistemática de Dicotiledôneas. Viçosa, MG, UFV.

Belgrano MJ, Pozner R. 2005. Stillingia yungasensis (Euphorbiaceae): A New Species from Northwestern Argentina and Southern Bolivia. Systematic Botany 30(1): 134138.

Bentham G, Hooker JD. 1880. Genera Plantarum 3(1). Lovell Reeve, London. UK. $685 \mathrm{p}$.

Corner EJH. 1976. The seeds of the dicotyledons. 2 vols. Cambridge University Press, Cambridge.

Esser HJ. 1993. Dendrothrix, a new generic concept in neotropical Euphorbiaceae. Novon 3: 245-251.

Esser HJ. 1994. Systematische studien an den Hippomaneae Adr. Juss. ex Bartling (Euphorbiaceae), insbesondere den Mabeinae Pax \& K. Hoffm. Dissertation zur Erlangung des Doktorgrades des Fachbereichs Biologie der Universität Hamburg, Hamburg, Germany.

Esser HJ. 1995. A taxonomic revision of Senefelderopsis Steyerm. (Euphorbiaceae), including additional notes on Dendrothix Esser. Mitteilungen aus dem Institut für Allgemeine Botanik in Hamburg 25: 121-133.

Esser HJ. 1997. A revision of Omalanthus (Euphorbiaceae) in Malesia. Blumea 42: 421-466.

Esser HJ. 1998. New combinations in Microstachys (Euphorbiaceae). Kew Bulletin 53(4): 955-960.

Esser HJ. 1999a. A partial revision of the Hippomaneae (Euphorbiaceae) in Malesia. Blumea 44: 149-215. 
Esser HJ. 1999b. Rhodothyrsus, a new genus of Euphorbiaceae from tropical South America. Brittonia 51(2): 170-180.

Esser HJ. 2001. Tribes Hippomaneae, Pachystromateae e Hureae; pp. 352-370. In: Radcliffe-Smith, A. (Ed.) Genera Euphorbiacearum, Royal Botanic Gardens, Kew, England.

Esser HJ. 2002. A revision of Triadica Lour. (Euphorbiaceae). Harvard Papers in Botany 7(1): 17-21.

Esser HJ. 2003. Fruit characters in Malesian Euphorbiaceae. Telopea 10(1): 2003.

Esser HJ, Welzen P, Djarwaningsh T. 1997. A phylogenetic classification of the Malesian Hippomaneae (Euphorbiaceae). Systematic Botany 22(4): 617-628.

Ferreira RA, BotelhoSA, DavideCA, Malavasi CA. 2001. Morfologia de frutos, sementes, plântulas e plantas jovens de Dimorphandra mollis Benth. - faveira (LeguminosaeCaesalpinioideae). Revista Brasileira de Botânica 24(3): 303-309.

Govaerts R, Frodin DG, Radcliffe-Smith A. 200o. World checklist and bibliography of Euphorbiaceae (with Pandaceae). 4 vols. Royal Botanic Gardens, Kew, UK.

Jablonski E. 1969. Notes on Neotropical Euphorbiaceae: 4. Monograph of the genus Actinostemon. Phytologia 18(4): 213-240.

Jussieu A. 1824. De Euphorbiacearum generibus medicisque earumdem viribis tentamen. Didot, Paris, France.

Kapil RN, Bor J, Bouman F. 1980. Seed appendages in Angiosperms. Botanische Jahrbücher für Systematik, Pflanzengeschichte und Pflanzengeographie 101(4): $555^{-573 .}$

Klotzsch JF. 1841. Neue weniger gekannte südamerikanische, Euphorbiaceen. Gattungen Archiv Für Naturgeschicthe 7: 190-260.

Kruijt RCA. 1996. A taxonomic monograph of Sapium Jacq., Anomostachys (Baill.) Hurus, Duvigneaudia J. Léonard and Sclerocroton Hochst. (Euphorbiaceae - tribe Hippomaneae). Bibliotheca Botanica 146: 1-109.

Melo AL. 2006. Revisão de Sebastiania Spreng. sensu stricto (Euphorbiaceae Hippomaneae). (Tese de Doutorado). Universidade Federal Rural de Pernambuco, Recife, PE. 
Mori SA, Silva LAM, Lisboa G, Corandin L. 1989. Manual de manejo do herbário fanerogâmico. Centro de Pesquisa do Cacau, Ilhéus. 103 p.

Müller J. 1863. Euphorbiaceae. Linnaea 32: 1-126.

Müller J. 1866. Euphorbiaceae. In: De Candolle, AP. (Eds.) Prodromus Systematis Naturalis Regni Vegetabilis 15(2): 189-1273.

Müller J. 1873. Euphorbiaceae. In: Martius, CFP. (Ed.). Flora Brasiliensis 11(2): 1-751.

Niembro A. 1988. Semillas de árboles y arbustos. Ontogenia y Estructura. Limusa, México, $285 \mathrm{p}$.

Oliveira DMT. 2001. Morfologia comparada de plântulas e plantas jovens de leguminosas em arbóreas nativas: espécies de Phaseoleae, Sophoreae, Swartzieae e Tephrosieae. Revista Brasileira de Botânica 24(1): 85-97.

Pax F, Hoffmann K. 1912. Euphorbiaceae-Hippomaneae. In: Engler, A. (Ed.) Das Pflanzenreich regni vegetabilis conspectus 4(147): 1-319.

Radford AE, Dickson WC, Massey JR. 1974. Vascular plant systematics. New York, Happer \& Row Publishers. 891 p.

Rogers DJ. 1951. A revision of Stillingia in the New World. Annals of the Missouri Botanical Garden 38: 207-259.

Roth I. 1977. Fruits of Angiosperms. In: K. Linsbauer (Ed.). Encyclopedia of plant anatomy 10: 148-194, Berlin: Gebrüder Borntraeger,

Santiago EF, Paoli AAS. 1999. Morfologia do fruto e da semente de Peltophorum dubium (Spreng.) Taubert (Leg-Caesalpinoideae). Naturalia 24: 139-152.

Santos VJ, Sales MF. 2009. A tribo Hippomaneae A. Juss. ex Spach. (Euphorbiaceae Juss.) no Estado de Pernambuco, Brasil. Acta Botanica Brasílica 23(4): 976-99o.

Scholz H. 1964. Euphorbiaceae. In: Engler, A. (Ed.) Syllabus der Pflanzenfamilien. Revised by H. Melchior.

Simpson MG. 2006. Plant Systematics. Elsevier Academic Press. 590 p.

Thiers B. 2017 [continuamente atualizado] Index Herbariorum: A global directory of public herbaria and associated staff. New York Botanical Garden's Virtual Herbarium, New York. Available from: http://sweetgum.nybg.org/ih/ (accessed 28 August 2016) 
Webster GL. 1994. Synopsis of the genera and suprageneric taxa of Euphorbiaceae. Annals of the Missouri Botanical Garden 8: 33-144.

Webster GL, Huft MJ. 1988. Revised synopsis of Panamanian Euphorbiaceae. Annals of the Missouri Botanical Garden 75: 1087-1144.

Werker E. 1997. Seed Anatomy. Berlin. 424 p. 